\title{
Otimização dos flutuadores de risers com catenária em configuração complexa
}

\section{Optimization of complex configuration of catenary risers buoyancy modules}

\author{
Marcos Diego S. V. Arruda ${ }^{\text {; }}$ Michele A. L. Martins ${ }^{2}$; Eduardo N. Lages ${ }^{3}$
}

\section{Resumo}

Em projetos de risers, dutos que conduzem o óleo e gás do poço à unidade de produção, a metodologia tradicional de pré-dimensionamento é baseada em tentativa e erro, o que proporciona a essa atividade uma considerável dependência da experiência do projetista. A utilização de risers com catenária em configuração complexa, capazes de permitir a extração de petróleo em condições de mar mais severas, pode agregar maior complexidade a esse processo de pré-dimensionamento, uma vez que novas variáveis de projeto são adicionadas ao problema pelo uso de módulos flutuadores acoplados em um segmento intermediário do riser. Este trabalho propõe a aplicação de uma metodologia de otimização dos flutuadores no pré-dimensionamento de risers com catenária em configuração complexa na determinação da configuração de equilíbrio estático. Para tanto, o problema de otimização é formulado matematicamente, resultando em um aplicativo computacional que combina o framework DOOLINES, um simulador para a análise estática e dinâmica de linhas de ancoragem e risers, e da toolbox de otimização do MATLAB ${ }^{\circledR}$, para o gerenciamento desse processo que faz uso de uma técnica híbrida: iniciando com Algoritmos Genéticos e finalizando com Programação Quadrática Sequencial. Um estudo de caso é apresentado e discutido, demonstrando a funcionalidade desse aplicativo que proporciona um resultado tido como o melhor possível, para a medida de eficiência adotada.

Palavras-chave: Otimização estrutural. Risers com catenária em configuração complexa. Algoritmo Genético. Programação Quadrática Sequencial.

\begin{abstract}
Risers are pipes that conduct oil and gas from the subsea wellhead to the floating production unit. For such structures, the pre-sizing approach is usually based on a trial-and-error methodology, fairly dependent on the designer's experience. The use of catenary risers in complex configuration brings the advantage of overcoming the challenges imposed by deep-water fields for oil exploitation. On the other hand, pre-sizing complexity increases as new design variables are added to define the geometrical parameters of the buoyancy modules attached to a riser intermediary segment. This work presents the pre-sizing approach of catenary risers in complex configuration using optimization techniques for the definition of the riser static equilibrium configuration. Thus, the optimization problem is numerically
\end{abstract}

\footnotetext{
${ }^{1}$ Mestrando do Programa de Pós-Graduação em Engenharia Civil da Universidade Federal de Alagoas; diegoarruda@1ccv.ufal.br.

2 Pesquisadora do Laboratório de Computação Científica e Visualização da Universidade Federal de Alagoas; micheleagra@lccv. ufal.br.

3 Professor Associado do Centro de Tecnologia da Universidade Federal de Alagoas; enl@lccv.ufal.br.
} 
represented and formulated resulting in a computational application that couples the framework DOOLINES, a simulator for the static and dynamic analysis of mooring lines and risers, with the MATLAB ${ }^{\circledR}$ optimization toolbox, for managing this process, applying a hybrid method, starting with the application of Genetic Algorithms followed by the Sequential Quadratic Programming. A case study is presented and discussed to demonstrate the functionality of this applicative that produces the optimal result according to an objective performance measure.

Key words: Structural optimization. Catenary riser in complex configuration. Genetic Algorithm. Sequential Quadratic Programming.

\section{Introdução}

Risers são estruturas tubulares consideradas esbeltas pela baixa razão entre o diâmetro e o comprimento. Quanto ao material constituinte, em geral, há dois tipos de risers: os rígidos e os flexíveis. Os risers rígidos, geralmente em aço, são compostos por tubos soldados. Os risers flexíveis são produzidos a partir do entrelaçamento de materiais poliméricos, que conferem isolamento e proteção, e de armaduras, que lhes garantem resistência mecânica.

Quanto à configuração geométrica, os risers flexíveis podem apresentar-se na forma de catenária livre e catenária complexa. Já os risers rígidos, além das configurações possíveis para as linhas flexíveis, podem apresentar a configuração de riser tensionado no topo (Top Tensioned Riser - TTR), ou vertical, e algumas configurações mais complexas que utilizam boias e tendões (CHAKRABARTI, 2005), como ilustrado na Figura 1.

Figura 1 - Configurações de riser: (a) vertical, (b) em catenária e (c) e (d) em catenária com configuração complexa.

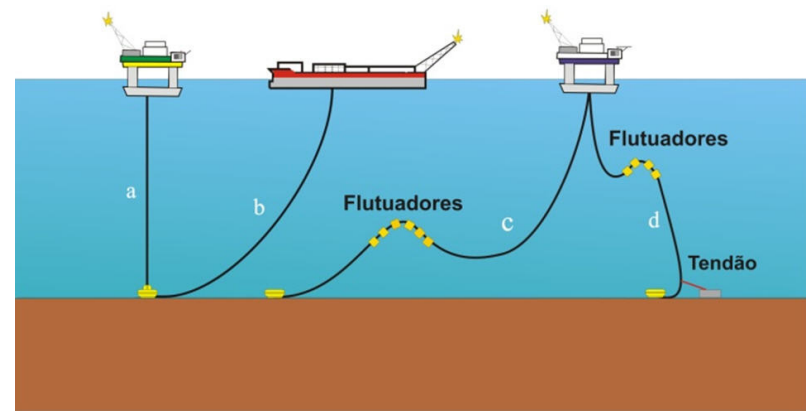

Fonte: Autor
A configuração do riser em catenária, por não ter que permanecer retilíneo entre suas conexões (caso da geometria vertical), torna-o capaz de melhor absorver a movimentação da plataforma sem comprometer sua integridade física, dada a sua complacência. Os risers complacentes experimentam carregamentos de onda, vento e corrente em condições mais severas - cenário de campos cada vez mais profundos e distantes do continente, como na região do pré-sal brasileiro.

Por outro lado, com o aumento da profundidade de exploração, alguns problemas surgem e podem inviabilizar a utilização de risers em catenária simples. Os principais problemas estão relacionados ao excesso de tração no topo, uma vez que todo o comprimento suspenso do riser é sustentado pelo ponto de conexão com a plataforma, e à baixa vida útil à fadiga, principalmente na conexão de topo e no ponto de contato com o solo (touch down pointTDP) (BELL; CHIN; HANRAHAN, 2005).

Para solucionar esses problemas, uma das alternativas para a configuração do riser em catenária é sua forma complexa (BAI, 2001). Sua geometria é adquirida, por exemplo, pela adoção de um conjunto de módulos flutuadores, dispostos em uma região intermediária da linha, que pelo empuxo atuante traz benefícios à dinâmica da estrutura (Figura 2). 
Figura 2 - Configuração de riser em catenária com configuração complexa através da adoção de flutuadores.

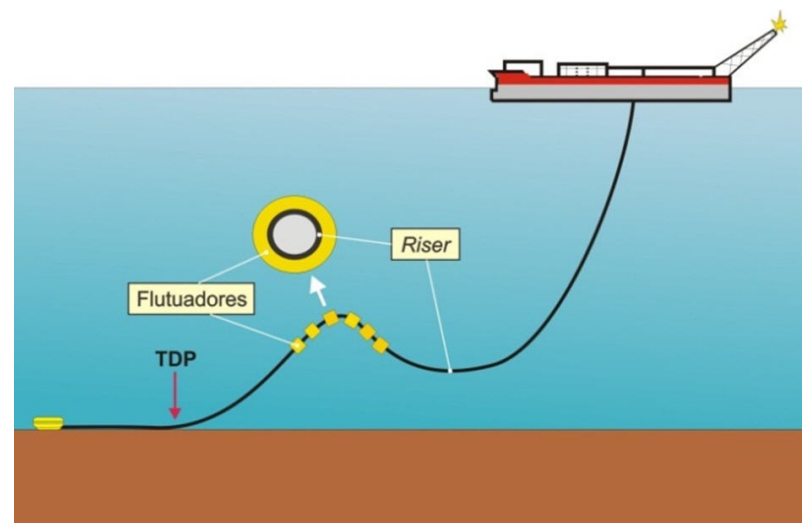

Fonte: Autor

Quanto ao dimensionamento de risers, esse deve ser realizado de modo a garantir os requisitos funcionais devidos aos carregamentos correspondentes ao meio interno (fluido transportado), ao meio externo, às cargas ambientais provenientes de ondas e correntes e movimentos da unidade flutuante durante a vida útil de projeto. Procedimentos para a adequada realização desse dimensionamento encontram-se regulamentados por normas internacionais, a exemplo da API-RP2RD (AMERICAN PETROLEUM INSTITUTE, 1998) e da DNV-OS-F201 (DET NORSKE VERITAS, 2001).

Froufe (2006) fez uma análise comparativa de critérios de dimensionamento de risers com base nas recomendações apresentadas pelas normas ABS (AMERICAN BUREAU OF SHIPPING, 2005), DNV (DET NORSKE VERITAS, 2001) e API (AMERICAN PETROLEUM INSTITUTE, 1999). Observou-se que cada norma apresenta suas considerações de carregamento de forma diferente, mas são basicamente equivalentes, levando-se em consideração cargas estáticas e dinâmicas no projeto dos risers. Além disso, ressaltou ainda que a filosofia entre elas é equivalente, embora as formulações não sejam as mesmas.
A geometria diferenciada característica do riser em catenária com configuração complexa agrega uma maior dificuldade no pré-dimensionamento desse tipo de estrutura, uma vez que o mesmo passa a depender da definição de variáveis de projeto adicionais relacionadas aos flutuadores, a exemplo do comprimento do segmento, do diâmetro dos módulos, do espaçamento entre os módulos, da densidade do material e do posicionamento desses dispositivos ao longo do riser. Seguindo uma metodologia tradicional de projeto, seriam analisadas configurações formadas a partir de diferentes combinações dessas e de outras variáveis de projeto, por tentativa e erro, até que se alcançasse uma configuração viável técnica e economicamente.

Devido à grande quantidade de variáveis envolvidas no projeto conceitual desse tipo de estrutura, são inúmeras as possibilidades de configurações possíveis, tornando a metodologia tradicional de projeto ineficiente e podendo conduzir fatalmente a projetos inviáveis. Nesse contexto, a utilização de uma metodologia de otimização no pré-dimensionamento desse tipo de riser surge como uma alternativa mais apropriada, capaz de conduzir a uma configuração geométrica que, além de viável, é ótima segundo a medida de eficiência desejada.

Este trabalho tem como objetivo empregar uma metodologia para o pré-dimensionamento de risers em catenária com configuração complexa aplicando otimização híbrida, combinando-se Algoritmos Genéticos e Programação Quadrática Sequencial. São consideradas apenas restrições com base na configuração de equilíbrio estático. Essa configuração ótima é admitida como condição inicial para a análise dinâmica ainda prevista na metodologia de projeto de risers. Como ferramenta numérica para experimentação dessa metodologia, é desenvolvido um aplicativo computacional que combina o framework DOOLINES (SILVEIRA; LAGES; FERREIRA, 2012) - para a análise estática do riser - e a toolbox de otimização do MATLAB ${ }^{\circ}$. 


\section{Referencial Teórico}

Em projetos conceituais de risers, com a motivação de superar os complexos problemas de dimensionamento com relativa rapidez e eficiência, alguns estudos já foram desenvolvidos abordando a aplicação de técnicas de otimização na prática de projeto.

Vieira (2008) abordou o dimensionamento de um riser em catenária lazy-wave, configuração que também adota um trecho flutuado, utilizando a metodologia de otimização por algoritmos genéticos. A ferramenta computacional desenvolvida foi aplicada em três etapas: uma avaliando uma configuração pré-definida aos limites de aplicação em um determinado cenário, outra para definir os materiais empregados em uma determinada configuração e, a última, incluindo a análise dinâmica da linha. A função objetivo utilizada foi o custo dos componentes do riser, tendo como restrições o ângulo no topo, tração e tensões ao longo do riser, as quais receberam a denominação de restrições de comportamento.

Vieira (2009) apresentou um estudo comparativo sobre métodos de otimização bio-inspirados aplicados a risers em catenária. Para isso foi desenvolvida uma ferramenta computacional que possibilitou o estudo empregando-se três métodos: Sistema Imunológico Artificial (SIA), Algoritmos Genéticos (AG) e Enxame de Partículas (Particle Swarm Optmization- PSO). O SIA apresentou melhores resultados em termos do número de avaliações necessárias para a convergência do processo de otimização. A função objetivo adotada também foi representativa do custo do material do riser, buscando-se minimizar o volume de flutuador necessário para atender às restrições impostas, semelhante ao trabalho supracitado.

A aplicação de risers em catenária lazywave conectadas a uma unidade de produção do tipo FPSO (Floating, Production, Storage and Offloading), que apresenta maior capacidade operacional na explotação em águas ultraprofundas, foi abordada por Andrade et al. (2010). Nesse estudo foram analisadas todas as etapas do processo de dimensionamento: análise de carregamento extremo, de instalação, de interferência e de fadiga. A otimização permitiu a diminuição do tempo total de projeto, pois diminuiu o número de ciclos demandados pela metodologia tradicional.

Tanaka e Martins (2011) também trabalharam com otimização de risers rígidos com foco em catenárias lazy-wave. Nesse estudo foram comparadas diferentes funções objetivo, das quais a minimização da máxima amplitude de tensão dinâmica apresentou melhores resultados, apesar de todas as demais apresentarem configurações viáveis: minimização da máxima tensão estática ao longo do riser e minimização do custo do material. Foram adotadas como restrições: raio de curvatura mínimo, tração mínima e máxima ao longo do riser, ângulo entre o riser e a vertical no topo e tração máxima nesse mesmo ponto (motivada pela capacidade de carga da unidade flutuante).

\section{Aspectos de Otimização}

\section{Estrutura de um problema de otimização}

Otimização é a ação de se obter o melhor resultado para um determinado problema sobre dadas circunstâncias. Com a modelagem de um problema desse tipo em termos de uma função matemática, dependente de variáveis de decisão, otimização pode ser definida como o processo de achar as condições que resultem no máximo ou mínimo valor para essa função (RAO, 2009).

A forma canônica de um problema de otimização consiste em

$$
\text { Achar arg min fobj }(\boldsymbol{X})
$$

tal que

$$
\begin{gathered}
g_{j}(\boldsymbol{X}) \leq 0 \text { para } j=1, \ldots, n_{g} \\
h_{k}(\boldsymbol{X})=0 \text { para } k=1, \ldots, n_{h} \\
\boldsymbol{l} \boldsymbol{b} \leq \boldsymbol{X} \leq \boldsymbol{u} \boldsymbol{b}
\end{gathered}
$$


onde $\boldsymbol{X}$ é o vetor que agrega as $n$ variáveis de decisão, $f o b j(\boldsymbol{X})$ é a função objetivo, $g_{j}(\boldsymbol{X})$ é a $j$-ésima restrição de desigualdade, $n_{g}$ é o número de restrições de desigualdade, $h_{k}(\boldsymbol{X})$ é $k$-ésima restrição de igualdade, $n_{h}$ é o número de restrições de igualdade e $\boldsymbol{l} \boldsymbol{b}$ e $\boldsymbol{u} \boldsymbol{b}$ são, respectivamente, os vetores dos limites inferior e superior da variável $\boldsymbol{X}$.

\section{Algoritmos Genéticos}

O desenvolvimento dos Algoritmos Genéticos (AG) surgiu da ideia de formular matematicamente a teoria de que indivíduos mais adaptados ao meio em que vivem possuem maior probabilidade de sobrevivência e, consequentemente, de reprodução (GOLDBERG, 1989; HOLLAND, 1975).

Esse mesmo processo acontece na solução de um problema de otimização com base em algoritmos genéticos. A cada passo, os algoritmos genéticos trabalham com um conjunto de soluções para o problema, que é denominado geração. Como se trata de um processo evolutivo, são selecionados aqueles indivíduos - no caso do presente trabalho, configurações de risers - que apresentam melhores resultados, para que esses originem descendentes de uma nova geração. Isso se repete até que um critério de parada é atingido. A aleatoriedade está presente nos passos dos algoritmos genéticos e é um de seus pontos fortes: sua capacidade de modificar de maneira aleatória algumas características de uma solução, em algumas etapas do processo, evita que a otimização fique restrita a um mínimo local.

\section{Programação Quadrática Sequencial}

A Programação Quadrática Sequencial (Sequential Quadratic Programming- SQP) é um dos mais recentes métodos de otimização desenvolvidos, como também um dos que apresentam melhores resultados (RAO, 2009).

Aplicada em problemas de otimização não lineares e com restrições, a SQP é considerada um método por aproximação, pois sua fundamentação consiste em aproximar a função objetivo por um modelo quadrático e linearizar as restrições não lineares. Dessa forma, o problema é transformado em um subproblema de programação quadrática, de mais simples resolução, e a direção de busca é dada pelo vetor gradiente.

\section{A Análise Estrutural}

\section{A análise do problema}

Em relação aos carregamentos atuantes, os risers são submetidos principalmente a ações resultantes da interação fluido-estrutura, como ações impostas por ondas e correntes marinhas, assim como o próprio movimento da plataforma a qual eles estão conectados. Também são considerados os esforços provocados pela força restauradora de contato com o solo e o peso próprio da estrutura (FERREIRA, 2005). No caso específico do presente trabalho, apenas os carregamentos estáticos são considerados, sendo eles: peso próprio, empuxo, corrente marinha e a interação com o solo.

No presente estudo, a avaliação estática do riser em catenária com configuração complexa considera que o mesmo consiste em uma estrutura com rigidezes axial e à flexão, porém livre à torção. Quanto às condições de contorno, o duto possui suas conexões fixas à translação, sendo que, na base, a conexão com o poço é livre à rotação e, no topo, a conexão com a unidade flutuante é realizada por meio de um apoio elástico.

\section{O framework DOOLINES}

O DOOLINES (Dynamics of Offshore Lines) é um framework orientado a objetos para análise não linear de estruturas marinhas esbeltas que apresentam o comportamento de cabos (SILVEIRA; LAGES; FERREIRA, 2012).

A característica de ser um framework indica que o DOOLINES é um sistema semicompleto, 
sendo necessária uma aplicação para lhe instanciar - alguém que o acione, tornando-o concreto, como é o caso do aplicativo desenvolvido neste trabalho. Alguns aplicativos já foram desenvolvidos com o DOOLINES, a exemplo do programa DYNASIM (NISHIMOTO; FUCATU; MASETTI, 2002), um simulador de estruturas offshore que analisa de forma integrada a unidade flutuante de produção, as linhas de ancoragem e os risers.

O DOOLINES possui como suporte numérico de discretização espacial do elemento estrutural, no caso as linhas de ancoragem e os risers, o Método dos Elementos Finitos (por exemplo ZIENKIEWICZ; TAYLOR, 2005), com a formulação proposta por Ghadimi (1988) para a descrição das forças internas em cada elemento finito.

Emprega-se a técnica de Relaxação Dinâmica (OTTER; DAY, 1960) para a análise estática não linear realizada pelo DOOLINES. De um modo geral, essa técnica consiste em resolver um problema estático transformando-o, artificialmente, em um problema dinâmico amortecido. A solução estática é a solução limite desse problema amortecido.

\section{Modelagem do Problema}

Neste trabalho, entende-se por modelagem a interpretação do problema real de um riser em catenária com configuração complexa, transformando-o em um modelo matemático de forma que possa ser trabalhado pelo aplicativo desenvolvido. São considerados aspectos tanto do problema de otimização quanto da representação numérica do riser para ser avaliado pelo DOOLINES.

\section{Representação numérica}

O riser em catenária com configuração complexa considerado neste trabalho é caracterizado pela presença de um segmento intermediário dotado de módulos flutuadores (Figura 3). É necessária, portanto, a definição de variáveis que determinem univocamente a forma (segmentos e materiais) de como o riser é composto.

A definição da configuração geométrica do modelo de riser considerado depende das dimensões dos diversos parâmetros que compõem o riser e o conjunto de módulos flutuadores: diâmetro interno (dint), diâmetro externo (dext), diâmetro dos módulos flutuadores (dextf), comprimento de cada módulo $(\mathrm{Lm})$, comprimento de arco inicial dos flutuadores $(L f i)$, comprimento de arco final dos flutuadores ( $L f f)$, comprimento total da linha $(L)$ e ângulo de topo do riser em relação à vertical $(\theta)$.

Adicionalmente, o ambiente de instalação fornece: projeção vertical $(L V)$, considerada a altura de lâmina d'água; projeção horizontal $(L H)$ e solo, considerado um plano horizontal.

Figura 3 - Parâmetros de projeto do modelo de riser em catenária com configuração complexa.

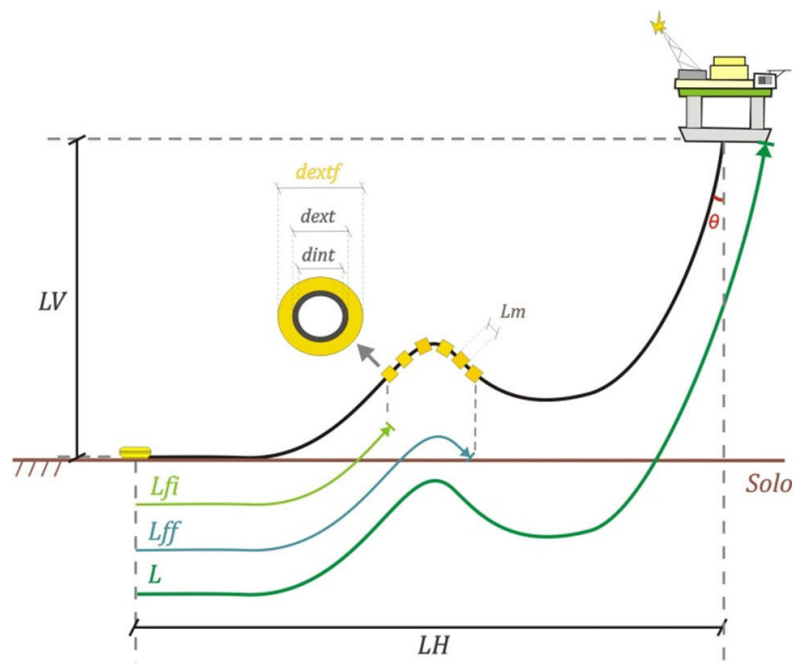

Fonte: Autor

\section{Variáveis de projeto}

Para um determinado local de instalação, o ideal para um trabalho de otimização seria deixar como variáveis de projeto todos os parâmetros geométricos e propriedades dos materiais do riser. Porém, segundo Tanaka (2009), o custo 
computacional para a otimização desse porte se tornaria demasiadamente grande. O aumento do tempo para o processo de otimização pode ser exponencial, prejudicando a velocidade da resposta. Por essa razão, é necessário estabelecer algumas simplificações para que o trabalho se realize de maneira satisfatória, deixando como variáveis de projeto aquelas de maior influência e fixando valores para as menos representativas.

Para este trabalho, são adotadas como variáveis de projeto a posição inicial dos flutuadores ( $L f i)$ e a posição final desses elementos ( $L f f)$. Essas variáveis implicam no posicionamento e no comprimento do conjunto de módulos flutuadores. Logo, o comprimento total do segmento de riser com flutuadores é dado pela diferença entre essas variáveis $(L f f-L f i)$ e a posição desse segmento ao longo do riser é definida a partir do comprimento de $\operatorname{arco}$ inicial $L f$.

\section{Função objetivo}

Diante de um problema de otimização de um riser em catenária com configuração complexa e definidas as variáveis de projeto como sendo $L f i$ e Lff, o objetivo é encontrar a melhor solução de maneira que essas variáveis impliquem em um resultado ótimo. A função objetivo adotada neste trabalho consiste no volume total de flutuador utilizado, que está relacionado proporcionalmente ao custo do mesmo. Minimizar o custo de material é uma forma bastante usual na otimização estrutural, e esse aspecto foi considerado nos trabalhos de Vieira (2008), Pina et al. (2008) e Vieira (2009) - que também tratam de otimização de risers. Portanto, a função objetivo é dada por

$$
f_{o b j}=(L f f-L f i)\left(\operatorname{dext} f^{2}-d e x t^{2}\right)
$$

Como o diâmetro externo do flutuador (dextf) e o diâmetro externo do riser (dext) são admitidos constantes, a função objetivo pode ser simplificada como

$$
f_{o b j}=L f f-L f i
$$

\section{Restrições}

Restrições são critérios de engenharia que delimitam os valores possíveis das variáveis de projeto de forma a tornar seus resultados mais próximos da realidade.

Inicialmente são definidas três restrições de natureza não linear, comumente encontradas na literatura. Duas são critérios de integridade estrutural, sendo a primeira a tensão máxima de serviço ao longo do riser (Tmax), que não pode ultrapassar a tensão máxima admissível do aço (Tadm), ou seja,

$$
\text { Tmax } \leq \text { Tadm }
$$

A segunda, também conhecida como payload, consiste na restrição quanto ao máximo esforço de tração que o riser pode exercer na conexão com a unidade flutuante (TracaoTopo), já que esta possui uma capacidade de carga máxima (TracaoTopomax), o que leva a

$$
\text { TracaoTopo } \leq \text { TracaoTopomax }
$$

A última restrição não linear é a cota mínima do vale do riser em relação ao solo (hvalemin). Essa restrição (Figura 4) é denominada altura do vale (hvale) e garante que a linha não entra em contato com o solo no trecho após os flutuadores, evitando assim um segundo ponto de contato com o solo (TDP). Portanto,

$$
\text { hvale } \geq \text { hvalemin }
$$

Figura 4 - Indicação da altura do vale.

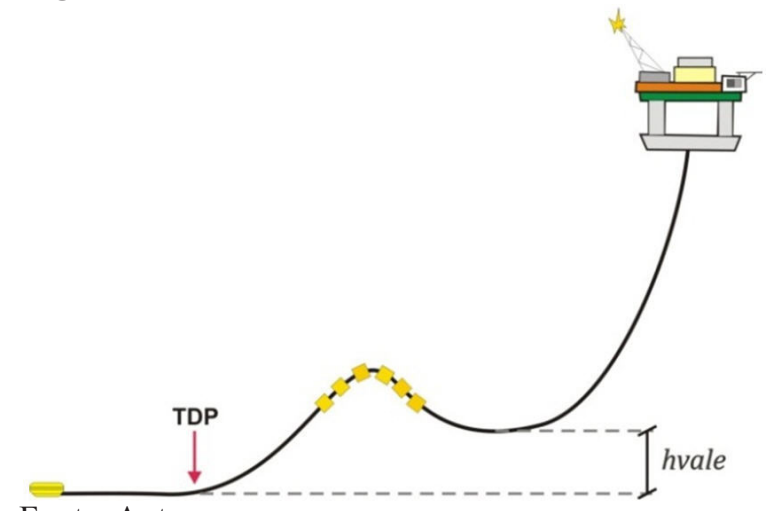

Fonte: Autor 
A partir da avaliação de exemplos preliminares de risers em catenária com configuração complexa, são incorporadas mais três restrições relevantes que não são diretamente abordadas na literatura.

Com base nessa avaliação, percebe-se que o posicionamento dos flutuadores muito próximo à conexão superior gera configurações inadequadas, nas quais o riser chega a atingir a superfície marítima. Esse tipo de situação deve ser evitado por razões técnicas (Figura 5).

Figura 5 - Riser em catenária com configuração complexa com flutuadores próximos do topo: configuração indesejável.

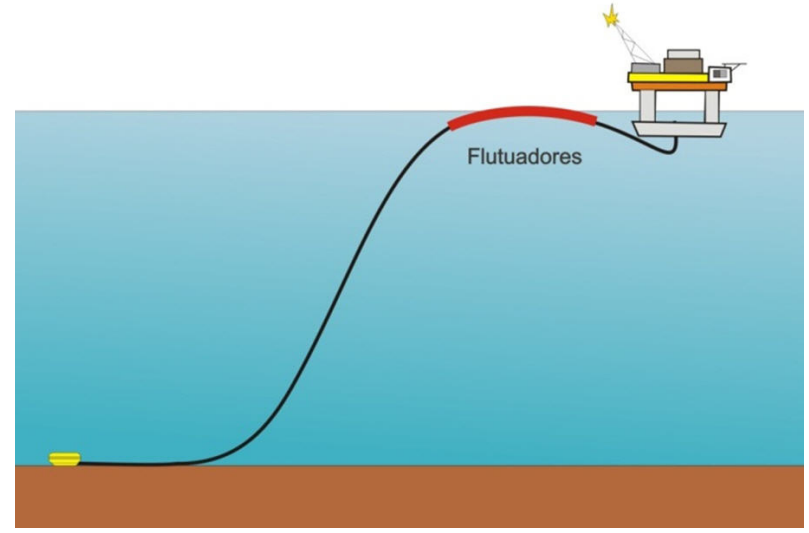

Fonte: Autor

Assim, é definido um valor percentual que, associado à altura de lâmina d'água, limita a profundidade mínima em que os flutuadores podem ser instalados, sendo, portanto, o limite superior das variáveis de projeto. Inicialmente esse valor é estabelecido em 50\%. Analogamente, o limite inferior definido corresponde ao comprimento de um elemento finito da malha (Lelem) para o posicionamento inicial $(L f i)$, obtendo-se a restrição

$$
\text { Lelem } \leq L f i \leq L-0,5 L V
$$

e de dois elementos para o posicionamento final (Lff) do trecho flutuado, ou seja,

$$
2 \text { Lelem } \leq L f f \leq(L-0,5 L V)+\text { Lelem }
$$

As simulações preliminares revelam ainda que o comprimento do trecho flutuado ótimo nunca atinge grandes valores. Assim, define-se também como restrição que o valor máximo que o comprimento dos flutuadores pode assumir é uma taxa de $20 \%$ do comprimento total do riser, ou seja,

$$
L f f-L f i \leq 0,2 L
$$

Se após simular um caso o valor ótimo encontrado estiver dentro desse intervalo, esse valor, necessariamente, é um ótimo global. Essa restrição promove eficácia no processo de otimização, pois evita o custo computacional da análise de modelos que não trarão um bom resultado.

Quanto às restrições lineares, neste trabalho elas são puramente geométricas. Além das apresentadas nas Eqs. 10, 11 e 12, há a exigência de que a posição inicial dos flutuadores deve ser menor que sua posição final, ou seja,

$$
L f i \leq L f f
$$

\section{O Aplicativo}

O aplicativo desenvolvido neste trabalho é composto pela incorporação do framework DOOLINES na toolbox de otimização do MATLAB $\AA$, e tem como objetivo encontrar uma configuração de riser com o menor volume possível de flutuador e que seja capaz de atender às restrições impostas. Para tanto, de acordo com a metodologia implementada, é feita uma busca por este resultado que consiste basicamente em propor soluções viáveis e, a partir de seus respectivos resultados, refiná-las tendo em vista a obtenção do resultado ótimo.

\section{As funções de otimização}

São utilizadas duas funções de otimização da toolbox do MATLAB ${ }^{\circledR}$ para esta aplicação: ga e fmincon, em uma abordagem conhecida como metodologia híbrida. A primeira encontra 
o mínimo de uma função através dos Algoritmos Genéticos, que é aplicada inicialmente no processo de otimização por possuir a capacidade de examinar todo o domínio de soluções possíveis, procurando assim pela região que apresenta melhores resultados a nível global. Após sua finalização, o resultado é refinado por meio da função fmincon, que tem a propriedade de apresentar um resultado ótimo com maior precisão, a partir do valor inicial obtido pela $g a$, aplicando o método SQP para a resolução de problemas de otimização não linear com restrições lineares e não lineares, de igualdade e desigualdade, a exemplo do problema apresentado neste trabalho.

\section{$A$ relação entre os sistemas $M A T L A B \circledR e$ DOOLINES}

A relação entre o MATLAB $\AA$, que gerencia o processo de otimização, e o DOOLINES, que realiza a análise estática de cada modelo, acontece da seguinte maneira: com os parâmetros que caracterizam a configuração da linha, gerados e fornecidos pelo aplicativo, o modelo é montado em um arquivo de entrada de dados para o simulador. Após a simulação da análise estática, um arquivo de saída é elaborado com as informações de interesse como tensão máxima de serviço ao longo do riser, tração no topo e dados necessários para o cálculo da altura do vale.

\section{O fluxo do aplicativo}

O procedimento inicial da aplicação é a definição dos parâmetros geométricos do problema, das propriedades dos materiais constituintes do riser e dos parâmetros de origem ambiental. Com essas informações é feita a modelagem do riser em catenária simples. Uma análise estática é realizada nesse tipo de configuração como forma de verificar se há a necessidade de lançar mão do riser em catenária com configuração complexa, ou seja, essa análise é realizada preliminarmente ao início do processo de otimização. Dessa forma, se a tensão máxima de serviço não ultrapassar a tensão admissível do material e a tração no topo for menor que o payload da unidade flutuante, não é necessária a utilização de flutuadores para minimizar esse efeito, assim, para as condições ambientais e de utilização do riser estabelecidas, a opção em catenária já atende aos critérios de projeto.

Caso o critério estrutural do riser não seja atendido pelo arranjo em catenária simples, o processo de otimização é iniciado: a função de otimização em uso propõe uma possível solução, essa é transformada em um modelo para ser analisado pelo DOOLINES e, com os resultados obtidos, a solução é avaliada quanto a viabilidade em relação às restrições impostas. Esse processo se repete até que um critério de parada seja atingido, ou seja, quando o resultado ótimo é encontrado.

\section{Estudo de caso}

Com a finalidade de demonstrar a funcionalidade da aplicação desenvolvida para otimização de risers em catenária com configuração complexa, um estudo de caso é apresentado.

\section{Dados de entrada}

\section{Dados do problema}

Os dados de entrada desta etapa são definidos com base na literatura, por meio de recomendações técnicas e visando um ambiente semelhante ao encontrado no pré-sal brasileiro. O comprimento total do riser $(L)$ é definido de forma que um riser modelado em catenária simples atenda à restrição do ângulo no topo recomendada: entre $5^{\circ}$ e $18^{\circ}$.

Dessa forma, os dados iniciais das informações referentes ao ambiente de instalação e ao riser são apresentados na Tabela 1. 


\section{Dados do processo de otimização}

Para o processo de otimização, realizado inicialmente por meio de um algoritmo genético através da função $g a$, são definidos os valores dos parâmetros desse método: função objetivo (Eq.6); número de variáveis de decisão (duas, Lfi e $L f f$ ); matriz e vetor representativos das restrições de desigualdade lineares (Eqs. 12 e 13); vetores representativos dos limites inferiores e superiores das variáveis de decisão (Eqs. 10 e 11); nome do arquivo com as restrições não lineares e nome da estrutura de opções de ga denominada gaoptimset, onde, por exemplo, são definidas as características dos operadores de seleção e reprodução.

A definição de alguns parâmetros de otimização é feita com base no estudo da influência de cada parâmetro dos algoritmos genéticos realizado por Tanaka (2009), a saber: número de gerações e de indivíduos, função de seleção, probabilidade de reprodução, número de indivíduos de elite, função de mutação e probabilidade de mutação. Os demais parâmetros do processo adotados são default de gaoptimset. Na Tabela 2 apresentam-se os referidos dados organizados segundo a estrutura da toolbox de otimização do MATLABß.

Tabela 1 - Dados para modelagem do riser.

\section{Dados geométricos}

\begin{tabular}{|c|c|c|}
\hline Projeção vertical do riser $-L V$ & 2200,0 & $\mathrm{~m}$ \\
\hline Projeção horizontal do riser - $L H$ & 4000,0 & $\mathrm{~m}$ \\
\hline Comprimento do riser $-L$ & 5200,0 & $\mathrm{~m}$ \\
\hline Diâmetro interno do riser - dint & 0,181 & $\mathrm{~m}$ \\
\hline Diâmetro externo do riser - dext & 0,219 & $\mathrm{~m}$ \\
\hline Comprimento do módulo flutuador - Lm & 0,50 & $\mathrm{~m}$ \\
\hline Diâmetro do módulo flutuador - dextf & 1,00 & $\mathrm{~m}$ \\
\hline \multicolumn{3}{|l|}{ Dados do material - aço X-52 } \\
\hline Peso específico - gaço & 78,5 & $\mathrm{kN} / \mathrm{m}^{3}$ \\
\hline Módulo de Young - MY & 210000000 & $\mathrm{kN} / \mathrm{m}^{2}$ \\
\hline Tensão de escoamento - $f y$ & 359000 & $\mathrm{kN} / \mathrm{m}^{2}$ \\
\hline Coeficiente de segurança & 1,5 & - \\
\hline Tensão admissível - Tadm & 239333,33 & $\mathrm{kN} / \mathrm{m}^{2}$ \\
\hline \multicolumn{3}{|c|}{ Dados do material - módulos flutuadores } \\
\hline Peso específico $-g f l u t$ & 5,529 & $\mathrm{kN} / \mathrm{m}^{3}$ \\
\hline \multicolumn{3}{|c|}{ Dado para definição dos segmentos } \\
\hline Comprimento do elemento - Lelem & 100,0 & $\mathrm{~m}$ \\
\hline \multicolumn{3}{|c|}{ Dados ambientais - perfil da corrente marítima } \\
\hline Profundidade (m) & $\begin{array}{l}\text { Velocidade } \\
(\mathrm{m} / \mathrm{s})\end{array}$ & Ângulo \\
\hline 0,0 & 2,0 & 0,0 \\
\hline 2200,0 & 0,0 & 0,0 \\
\hline
\end{tabular}

Fonte: Dados do autor 
Tabela 2 - Dados de entrada da função de otimização ga via gaoptimset.

\begin{tabular}{|c|c|c|}
\hline Parâmetro & Descrição & Valor \\
\hline CreationFcn & População inicial & gacreationuniform \\
\hline CrossoverFcn & Função que realiza o cruzamento & crossoverscattered \\
\hline CrossoverFraction & Probabilidade de reprodução & 0,9 \\
\hline DistanceMeasureFcn & Função de medida da distância de indivíduos & phenotype \\
\hline EliteCount & Número de indivíduos de elite & 2 \\
\hline FitnessLimit & Valor limite de aptidão & -inf \\
\hline FitnessScalingFcn & Função que escala os valores da função de aptidão & fitscalingrank \\
\hline Generations & Número de gerações & 30 \\
\hline HybridFcn & Função de otimização após o término de $g a$ & fmincon \\
\hline InitialPenalty & Penalidade inicial & 10 \\
\hline MigrationDirection & Direção da migração & forward \\
\hline MigrationFraction & Fração de indivíduos que migram & 0,2 \\
\hline MutationFcn & Função de mutação & fmutationuniform \\
\hline PenaltyFcn & Fator de atualização de penalidade & 100 \\
\hline PopInitRange & Variação da população inicial & {$[0 ; 1]$} \\
\hline PopulationSize & Tamanho da população & 50 \\
\hline SelectionFcn & Função de seleção & selectionroulette \\
\hline PenaltyFcn & Fator de atualização de penalidade & 100 \\
\hline UseParallel & Processamento paralelo & always \\
\hline TolFun & Tolerância para não melhoramento da função objetivo & $10^{-6}$ \\
\hline
\end{tabular}

Fonte: Dados do autor

\section{Resultados}

Comentários sobre o processo de otimização

A Figura 6 apresenta o histórico do valor da função objetivo (Eq. 6) durante o processo híbrido de otimização. Verifica-se que até a simulação 9369, as respostas obtidas oscilam significativamente o seu valor: esse comportamento se deve ao funcionamento do algoritmo genético pelos operadores de reprodução e mutação, que combinam e alteram, respectivamente, de forma aleatória os valores das variáveis na composição de um novo indivíduo. Após a finalização do algoritmo genético, o modelo otimizado apresenta um trecho flutuado de $400 \mathrm{~m}$. Quando o processo de otimização tem continuidade com a função fmincon, refinandose o resultado definido por ga, o comprimento do conjunto de flutuadores diminui para $300 \mathrm{~m}$ no riser otimizado. 
Ainda em referência à Figura 6, nota-se a ocorrência de valores negativos para a função objetivo, o que contraria a restrição geométrica que diz que o comprimento de arco inicial do trecho flutuado deve ser menor que o comprimento final desse trecho (Eq. 13). Essas ocorrências indicam que nem sempre a função de otimização ga propõe soluções que respeitam as restrições lineares, mas é certo que essas soluções são posteriormente avaliadas quanto à aptidão e, consequentemente, são associadas a um baixo valor desse parâmetro.
Ao longo do processo, é possível observar que há alguns patamares aos quais os valores da função objetivo parecem tender. No primeiro, o comprimento do trecho flutuado assume cerca de $1000 \mathrm{~m}$; a partir aproximadamente da simulação 6000, seu comprimento reduz para cerca de $400 \mathrm{~m}$ e, já no final, após um decrescimento nas últimas 800 simulações, a função objetivo oscila em torno de $300 \mathrm{~m}$. Assim, observa-se que o algoritmo de otimização trabalha no sentido de sempre reduzir o volume total de flutuadores - conforme esperado.

Figura 6 - Evolução da função objetivo ao longo do processo de otimização.

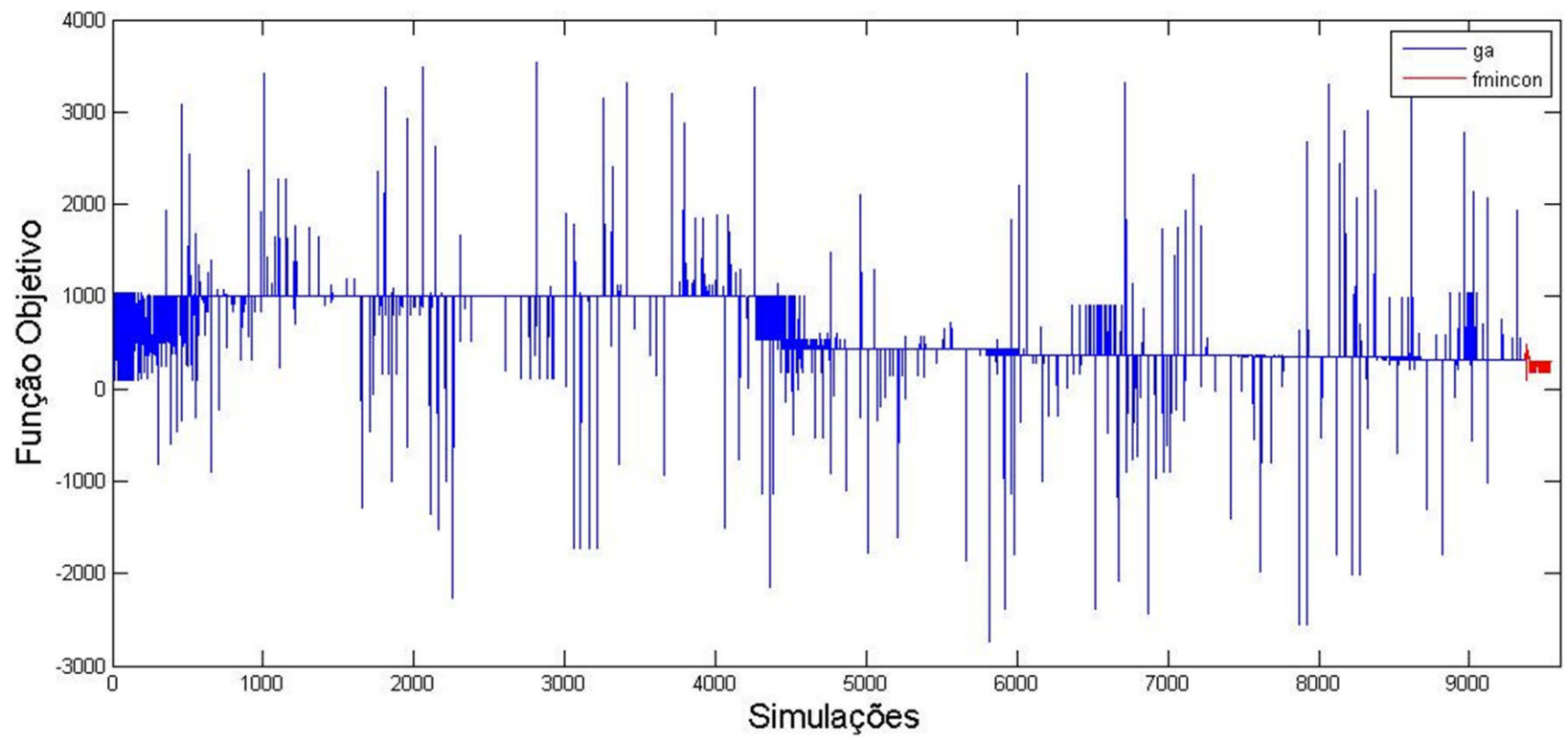

Fonte: Dados do autor

Para avaliar as restrições e verificar quais são mais difíceis de serem atendidas, contabiliza-se a violação de cada uma delas ao longo do processo. Os resultados gerais obtidos seguem na Figura 7 e, sobre cada restrição individualmente, na Figura 8. É importante lembrar que todo modelo analisado estruturalmente - para serem avaliadas as restrições não lineares - atendeu previamente às restrições geométricas lineares.
Figura 7 - Quantidade de restrições atendidas e violadas.

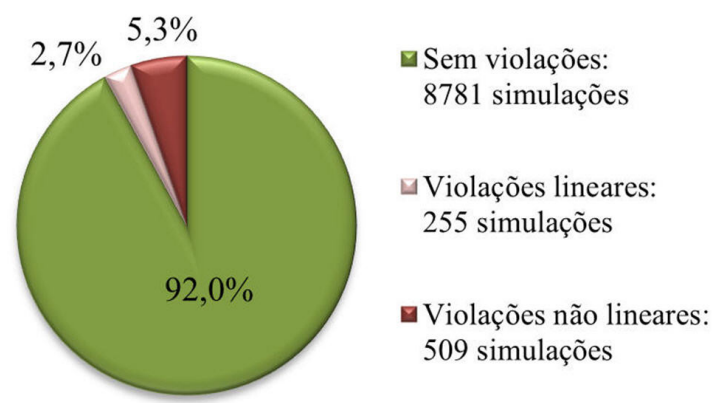

Fonte: Dados do autor 
De acordo com a Figura 7 observa-se que o processo de otimização propõe soluções nas quais a grande maioria atende a todos os critérios geométricos e estruturais: $92,0 \%$. Dos 8,0\% de modelos que violam alguma restrição, percebe-se que cerca de $1 / 3$ desses $(2,7 \%$ do total de simulações) não respeita restrições lineares e a maioria, cerca de $2 / 3(5,3 \%$ do total), viola as restrições não lineares. A minoria de violações lineares se justifica pelo fato de que o algoritmo é capaz de identificar com mais facilidade uma região que não produza resultados viáveis com base no histórico do processo de otimização.

$\mathrm{Na}$ Figura 8 são detalhadas as informações sobre as violações das restrições. Nas restrições lineares, o número de violações do critério $L f i \leq$ Lff (Eq. 13) é inferior à quantidade de indivíduos que violam o critério $L f f-L f i \leq 0,2 L$ (Eq. 12). Por serem responsáveis por apenas 2,7\% do número total de soluções avaliadas, pode-se dizer que essas restrições não geraram grandes dificuldades na busca pelo resultado ótimo.

Adicionalmente, percebe-se que a restrição não linear de tensão máxima de serviço (Eq. 7) é violada em uma quantidade um pouco inferior que o número de violações do critério de altura do vale (Eq. 9). Quanto ao critério de tração máxima no topo (Eq. 8), destaca-se que em todas as 509 violações de restrições não lineares essa condição não é atendida, sendo a de maior dificuldade para ser contornada.

Figura 8 - Violações das restrições não lineares e lineares.

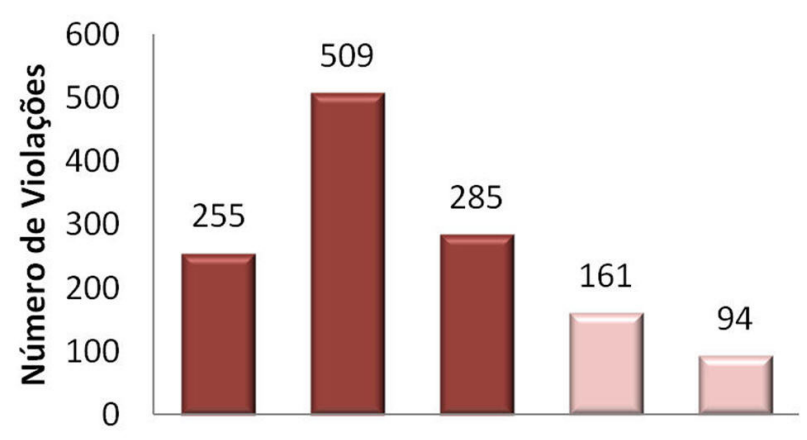

$\begin{array}{lllll}\text { Eq. } 7 & \text { Eq. } 8 & \text { Eq. } 9 & \text { Eq. } 12 & \text { Eq. } 13\end{array}$

Fonte: Dados do autor

\section{Análise do riser em catenária simples}

A primeira etapa do aplicativo desenvolvido é a verificação se um riser em catenária livre atende aos critérios de tensão máxima de serviço ao longo do riser, tração máxima na conexão superior e o ângulo entre essa e a vertical. A Figura 9 apresenta, na coloração azul, a configuração de equilíbrio deste riser e a Tabela 3 apresenta os resultados dessa análise preliminar.

Tabela 3 - Resultados da análise do riser em catenária simples.

\begin{tabular}{lcc}
\hline Comprimento do riser $-L$ & 5200 & $\mathrm{~m}$ \\
\hline Tensão máxima de serviço -Tmax & 182036,74 & $\mathrm{kN} / \mathrm{m}^{2}$ \\
Localização de Tmax & Topo & - \\
Tração no topo - TracaoTopo & 2144,53 & $\mathrm{kN}$ \\
Ângulo no topo - $\theta$ & 18,74 & Graus \\
\hline
\end{tabular}

Fonte: Dados do autor

Da Tabela 3, percebe-se que para esse caso específico o Tabela 4 - Resultados da análise do riser em catenária com configuração complexa otimizado em catenária livre respeita o critério da tensão máxima de serviço (menor que 239333,33 kN/m²), mas não consegue transmitir um esforço de tração menor que $1500 \mathrm{kN}$ para a unidade flutuante. Assim, torna-se justificável o uso de um trecho flutuado para que o empuxo atuante alivie esse esforço no topo. Quanto às conexões, tanto a da base quanto a do topo estão consoantes com os critérios técnicos: a inferior está na horizontal e a superior em um ângulo próximo de $18^{\circ}$.

\section{Análise do riser em catenária com configuração complexa otimizado}

Decorrido o processo de otimização, os parâmetros do riser em catenária com configuração complexa otimizado seguem na Tabela 4 e sua configuração de equilíbrio é apresentada na Figura 9. 
Tabela 4 - Resultados da análise do riser em catenária com configuração complexa otimizado

\begin{tabular}{lcr}
\hline Comprimento do riser $-L$ & 5200 & $\mathrm{~m}$ \\
Comprimento do trecho inicial & 3000 & $\mathrm{~m}$ \\
Comprimento do trecho flutuado & 300 & $\mathrm{~m}$ \\
Comprimento do trecho final & 1900 & $\mathrm{~m}$ \\
Tensão máxima de serviço-Tmax & $218565,45 \mathrm{kN} / \mathrm{m}^{2}$ \\
Localização de Tmax & Topo & - \\
Tração no topo - TracaoTopo & 1419,76 & $\mathrm{kN}$ \\
Altura do vale - hvale & 755,27 & $\mathrm{~m}$ \\
Ângulo no topo com a vertical $-\theta$ & 4,98 & graus \\
\hline
\end{tabular}

Fonte: Dados do autor

Os resultados do modelo ótimo observados na Tabela 4 confirmam que foi encontrado um riser que atende aos critérios estruturais: tensão máxima de serviço, apesar de superior ao modelo em catenária, inferior à admissível $\left(239333,33 \mathrm{kN} / \mathrm{m}^{2}\right)$; tração na conexão superior menor que $1500 \mathrm{kN}$ e altura do vale muito superior ao mínimo de $20 \mathrm{~m}$. Percebe-se também que o ângulo do topo com a vertical está dentro do intervalo mínimo recomendado (próximo de $5^{\circ}$ ). Embora esse quesito não tenha sido imposto como restrição, foi modelado com a finalidade de transmitir uma maior fidelidade ao problema real.

Os comprimentos ótimos dos segmentos também são apresentados e atendem às restrições geométricas (lineares) assim como os objetivos desse processo: o comprimento do trecho flutuado é de $300 \mathrm{~m}$ posicionado a partir do comprimento de arco de $3000 \mathrm{~m}$ partindo da base ao longo do riser.

A Figura 9 apresenta o riser otimizado, em verde com trecho flutuado em preto, e o modelo em catenária simples, em azul, para efeito de comparação.
Figura 9 - Riser em catenária com configuração complexa otimizado e riser em catenária simples do estudo de caso.

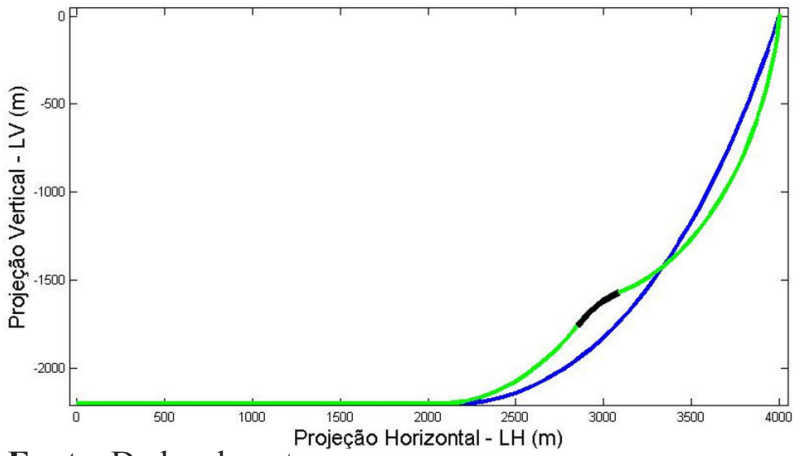

Fonte: Dados do autor

A configuração de equilíbrio do modelo otimizado apresenta-se coerente com os dados de resposta da aplicação (Tabela 4). Pela Figura 9 também é possível observar que o riser otimizado possui um comprimento apoiado no solo apenas um pouco inferior do que o riser em catenária simples e que o ângulo da conexão com a vertical diminuiu.

A localização do trecho flutuado está consoante com a seguinte ideia: o riser em catenária simples, a base do problema, possui um trecho suspenso e um trecho apoiado no solo; se no topo do riser é onde se localiza o ponto crítico da estrutura (maiores esforços de tração e tensão), então é justo que os flutuadores sejam dispostos na região suspensa a fim de provocar a diminuição dos esforços no topo, sendo necessário suspender um mínimo do comprimento apoiado por consequência. Dessa forma, é como se o problema se restringisse somente ao trecho suspenso.

\section{Conclusões}

O presente trabalho propôs a aplicação de técnicas de otimização no pré-dimensionamento dos módulos flutuadores de risers em catenária 
com configuração complexa. Esse propósito, como metodologia de projeto preliminar, decorre do fato de que um riser com essa configuração apresenta uma série de variáveis de projeto, dificultando a realização dessa atividade pelo projetista. $\mathrm{O}$ aplicativo de otimização desenvolvido e seus resultados apresentados, para um estudo de caso, atestam que essa metodologia é válida, chegando assim em melhores resultados de acordo com uma medida objetiva de eficiência e de maneira rápida e automática.

\section{Agradecimentos}

Os autores agradecem ao apoio financeiro da Agência Nacional do Petróleo, Gás Natural e Biocombustíveis - ANP -, da Financiadora de Estudos e Projetos - FINEP - e do Ministério da Ciência e Tecnologia-MCT - por meio do Programa de Recursos Humanos da ANP para o Setor Petróleo e Gás - PRH-ANP/MCT.

\section{Referências}

AMERICAN BUREAU OF SHIPPING. Guide for Building and Classing Subsea Riser Systems. Houston, 2005.

AMERICAN PETROLEUM INSTITUTE. API-RP-1111: design, construction, operation, and maintenance of offshore hydrocarbon pipelines (Limit State Design). Washington, 1999.

AMERICAN PETROLEUM INSTITUTE. API-RP-2RD: design of risers for floating production systems (FPSs) and tension-leg plataforms (TLPs). Washington, 1998.

ANDRADE, E. Q.; AGUIAR, L. L.; SENRA, S. F.; SIQUEIRA, E. F. N.; TORRES, A. L. F. L.; MOURELLE, M. M. Optimization procedure of steel lazy-wave riser configuration for spread moored FPSOs in deepwater offshore Brazil. In: OFFSHORE TECHNOLOGY CONFERENCE, 2010, Houston. Proceedings... Houston: Offshore Technology Conference, 2010.

BAI, Y. Pipeline and risers. Houston: American Bureau of Shipping, Elsevier, 2001. v. 3.

BELL, J. M.; CHIN, Y. D.; HANRAHAN, S. State- of-art of ultra deepwater production technologies. In: OFFSHORE TECHNOLOGY CONFERENCE, 2005, Houston. Proceedings... Houston: Offshore Technology Conference, 2005.

CHAKRABARTI, S. K. Handbook of offshore engineering. Amsterdam: Elsevier, 2005. v. 2.

DET NORSKE VERITAS. Offshore standard DNVOS-F201: dynamic risers. Hovik, 2001.

FERREIRA, F. M. G. Desenvolvimento e aplicações de um framework orientado a objetos para análise dinâmica de linhas de ancoragem e de risers. 2005. Dissertação (Mestrado em Engenharia Civil) - Universidade Federal de Alagoas, Maceió.

FROUFE, L. Análise Comparativa de Critérios de Dimensionamento de Risers Rígidos. 2006. Dissertação (Mestrado em Ciências em Engenharia Oceânica) Instituto Alberto Luiz Coimbra de Pós-Graduação e Pesquisa de Engenharia, Universidade Federal do Rio de Janeiro, Rio de Janeiro.

GHADIMI, R. A simple and efficient algorithm for the static and dynamic analysis of flexible marine risers. Computers and Structures, Elmsford, v. 28, n. 4, p. 541$555,1988$.

GOLDBERG, D. E. Genetic algorithms in search, optimization, and machine learning. Boston: AddisonWesley Pub, 1989.

HOLLAND, J. H. Adaptation in natural and artificial systems. Michigan: University of Michigan Press, 1975.

NISHIMOTO, K.; FUCATU, C. H.; MASETTI, I. Q. Dynasim: a time domain simulator of anchored FPSO. Journal of Offshore Mechanics and Artic Engineering, v. 124, n. 4, p. 203-211, 2002.

OTTER, J. R. H.; DAY, A. S. Tidal flow computations. The Engineer, London, v. 209, p. 177-182, 1960.

PINA, A. A.; LIMA, B. S. L. P.; ALBRECHT, C. H.; JACOB, B. P. Parameter selection and convergence analysis of the PSO algorithm applied to the design of risers. In: EngOpt - INTERNATIONAL CONFERENCE ON ENGINEERING OPTIMIZATION, 2008, Rio de Janeiro. Proceedings... Rio de Janeiro, 2008.

RAO, S. S. Engineering optimization: theory and practice. 4. ed. New Jersey: John Wiley \& Sons, 2009.

SILVEIRA, E. S. S.; LAGES, E. N.; FERREIRA, F. M. G. DOOLINES: an object-oriented framework for nonlinear static and dynamic analyses of offshore lines. Engineering with Computers, New York, v. 28, n. 2, p. 149-159, 2012. 
TANAKA, R. L. Otimização da configuração de risers rígidos. 2009. Tese (Doutorado em Engenharia de Controle e Automação Mecânica) - Escola Politécnica da Universidade de São Paulo, Departamento de Engenharia Mecatrônica e de Sistemas Mecânicos, São Paulo.

TANAKA, R. L.; MARTINS, C. A. Parallel dynamic optimization of steel risers. Journal of Offshore Mechanics and Arctic Engineering, New York, v. 133, n. $1,2011$.

VIEIRA, I. N. Algoritmos Bio-inspirados aplicados à otimização de risers rígidos em Catenária. 2009. Dissertação (Mestrado em Engenharia Civil) Universidade Federal do Rio de Janeiro, Rio de Janeiro.

VIEIRA, L. T. Otimização de sistemas de risers para explotação de petróleo offshore através de algoritmos genéticos paralelos. 2008. Tese (Doutorado em Engenharia Civil) - Universidade Federal do Rio de Janeiro, Rio de Janeiro.

ZIENKIEWICZ, O. C.; TAYLOR, R. L. The Finite Element Method, 6. ed. Oxford: Butterworth-Heinemann, 2005.

Recebido em 29 Março 2012-Received on March 29, 2012.

Aceito em 3 Julho, 2012 - Accepted on July 3, 2012. 